\title{
Coarse Grained Model for Semiquantitative Lipid Simulations
}

\author{
Siewert J. Marrink,* Alex H. de Vries, and Alan E. Mark \\ Department of Biophysical Chemistry, University of Groningen, Nijenborgh 4, \\ 9747 AG Groningen, The Netherlands
}

Received: August 22, 2003; In Final Form: October 29, 2003

\begin{abstract}
This paper describes the parametrization of a new coarse grained (CG) model for lipid and surfactant systems. Reduction of the number of degrees of freedom together with the use of short range potentials makes it computationally very efficient. Compared to atomistic models a gain of 3-4 orders of magnitude can be achieved. Micrometer length scales or millisecond time scales are therefore within reach. To encourage applications, the model is kept very simple. Only a small number of coarse grained atom types are defined, which interact using a few discrete levels of interaction. Despite the computational speed and the simplistic nature of the model, it proves to be both versatile in its applications and accurate in its predictions. We show that densities of liquid alkanes from decane up to eicosane can be reproduced to within $5 \%$, and the mutual solubilities of alkanes in water and water in alkanes can be reproduced within $0.5 k T$ of the experimental values. The CG model for dipalmitoylphosphatidylcholine (DPPC) is shown to aggregate spontaneously into a bilayer. Structural properties such as the area per headgroup and the phosphate-phosphate distance match the experimentally measured quantities closely. The same is true for elastic properties such as the bending modulus and the area compressibility, and dynamic properties such as the lipid lateral diffusion coefficient and the water permeation rate. The distribution of the individual lipid components along the bilayer normal is very similar to distributions obtained from atomistic simulations. Phospholipids with different headgroup (ethanolamine) or different tail lengths (lauroyl, stearoyl) or unsaturated tails (oleoyl) can also be modeled with the $\mathrm{CG}$ force field. The experimental area per headgroup can be reproduced for most lipids within 0.02 $\mathrm{nm}^{2}$. Finally, the CG model is applied to nonbilayer phases. Dodecylphosphocholine (DPC) aggregates into small micelles that are structurally very similar to ones modeled atomistically, and DOPE forms an inverted hexagonal phase with structural parameters in agreement with experimental data.
\end{abstract}

\section{Introduction}

Solutions of lipids and surfactants display a very rich phase behavior, including micellar, lamellar, hexagonal, and cubic phases. The structural characteristics of these phases and the details of the phase diagrams strongly depend on the physicochemical nature of the constituents. Understanding the relation between molecular structure and aggregation behavior is therefore of great importance. Computer simulations have provided a useful tool to elucidate the structure of lipid phases, especially the micellar and lamellar phases. Atomistic simulations reveal maximum detail but are restricted to small length and time scales. Although spontaneous aggregation processes can also be modeled atomistically (e.g., see ref 1), a more computationally efficient simulation model is required to fully investigate the rich phase behavior of lipid and surfactant solutions.

Coarse grained (CG) models, in which small groups of atoms are represented by single interaction sites, are becoming increasingly popular to study systems of lipids and surfactants (for recent reviews, see refs 2 and 3). CG models can be either on-lattice or off-lattice. Whereas the first is faster, the off-lattice type models are more versatile and realistic. The CG model that we present in this paper is an off-lattice model. The first off-lattice CG lipid model was developed by Smit et al. ${ }^{4}$ In their model two different interaction sites are distinguished: either water-like or oil-like. They interact via a Lennard-Jones type

* Corresponding author. E-mail: marrink@chem.rug.nl. interaction potential that is purely repulsive for water-oil interactions or short range attractive for like particles. Strings of these particles can successfully represent lipid and surfactant like molecules. Simulations based on the Smit model have subsequently been applied to study the formation and structure of micelles (e.g., see refs 5 and 6) and bilayers (e.g., see refs 7 and 8). Omitting explicit solvent in the simulation further increases the accessible length and time scales and allows stochastic simulations of the concentration dependent micelle aggregation $^{9}$ or vesicle formation, ${ }^{10}$ for instance. Another stochastic approach, which has its origin in simulations of block copolymers, ${ }^{11}$ is the method of dissipative particle dynamics (DPD). In DPD the lipids are modeled as soft beads, representing fluid elements rather than real particles. The beads interact pairwise via a combination of repulsive, dissipative, and random forces. As with the Smit model, springs define molecular architecture. The DPD technique has found recent applications in the area of lipids and surfactants, with simulations of bilayer structure, ${ }^{12}$ elastic properties, ${ }^{13}$ self-assembly, ${ }^{14}$ pore formation,,${ }^{15}$ vesicle formation and fusion, ${ }^{16}$ and the construction of a complete phase diagram for a simple $\mathrm{AB}$ type surfactant. ${ }^{17}$

Although the current CG models used in MD and DPD are becoming very powerful in understanding structural aspects of lipid and surfactant phases and the relative phase stabilities, the models cited above are qualitative rather than quantitative in their predictions. Illustrative of this fact is the use of dimensionless units to measure the length, time, and energy scales. Where a link is made to realistic systems the mapping onto physical measures is done in hindsight. The question is, can a CG type 
model be specifically parametrized to model a realistic lipid system in advance and can it then be used to make quantitative predictions? A recent attempt to do so was made by Shelley et al. ${ }^{18}$ They parametrized a CG model to explicitly model DMPC. LJ interactions for solvent sites and tail groups are optimized to reproduce the bulk properties of water and alkanes. Atomistic simulations of DMPC bilayers are used to optimize the parameters for the lipid headgroup and the angle potentials of the lipid tails. Electrostatic interactions are also taken into account explicitly in this model. Monte Carlo and MD simulations with this model show the assembly of DMPC into bilayers with properties in semiquantitative agreement with atomistic results. ${ }^{18}$ Although suggesting that more quantitative results could be obtained with CG models, the Shelley model has several limiting features: it is optimized for a particular lipid in a particular phase only, it uses complicated interaction functions requiring short integration time steps, and it evaluates long-range interactions.

To improve on the CG models currently available, four aspects are deemed important: speed, accuracy, applicability, and versatility. In the CG model presented in this paper these four aspects are optimized simultaneously: (i) Speed is obtained by including only short-range interactions and by the use of smooth potentials such that large integration steps can be used. (ii) Accuracy is maximized by matching CG results to atomistic simulations as much as possible, for a variety of components and phases simultaneously. In this process structural and dynamical as well as thermodynamical data are used. (iii) Applicability is enhanced through the simplicity of the force field, the use of standard interaction potentials, and few parameters. Furthermore, the parameters are physically meaningful and used in a consistent manner. (iv) Versatility is implied as the force field leaves enough room to accommodate structural detail of molecules, and because there is no restriction to the phase of the system.

The rest of this paper is organized as follows. An extensive description of the model is presented in the next section. Applications of the model are then shown for a number of systems: alkane/water, a salt solution, lipid bilayers, and nonbilayer phases. A short discussion follows in which the merits and shortcomings of the model are examined.

\section{Model}

2.1. Interaction Sites. A four-to-one mapping is used to represent the molecules in the simplified model; i.e., on average, four atoms are represented by a single interaction center. This rule is not strict, as sometimes it is appropriate to map three, five, or more atoms into one interaction center. Because of their small size and mass, hydrogen atoms are not considered at all. To keep the model simple, we currently consider four main types of interaction sites only: polar (P), nonpolar $(\mathrm{N})$, apolar $(\mathrm{C})$, and charged (Q). Polar sites represent neutral groups of atoms that would easily dissolve into water (e.g., ethylene glycol), apolar sites represent hydrophobic moieties (e.g., butane), and nonpolar groups are used for mixed groups which are partly polar, partly apolar (e.g., propanol). Charged sites are reserved for ionized groups (e.g., ammonium). For particles of type $\mathrm{N}$ and Q four subtypes ( $0, \mathrm{~d}, \mathrm{a}$, and da) are further distinguished. The subtypes allow fine-tuning of the interactions on the basis of the chemical nature of the atoms, which are represented by the CG groups. Subtype 0 applies to groups in which no hydrogen bonding capabilities exist, $\mathrm{d}$ and a to groups that could act as a hydrogen bond donor or acceptor, respectively, and da to groups with both donor and acceptor options.

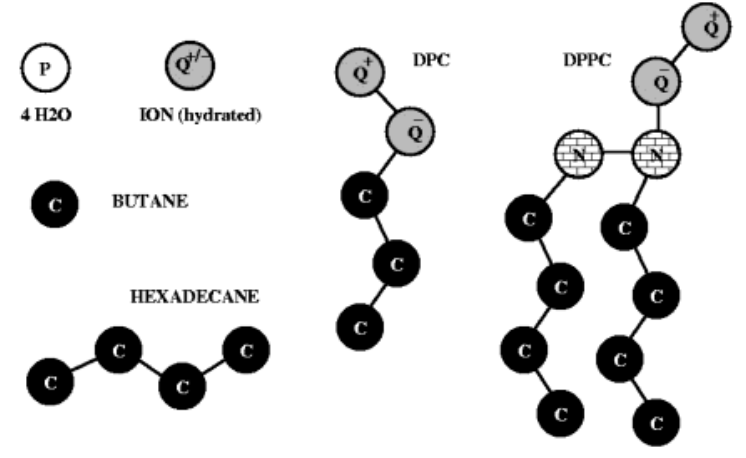

Figure 1. Mapping in the coarse grained model for water, ions, butane, hexadecane, DPC (dodecylphosphocholine), and DPPC (dipalmitoylphosphatidylcholine). All atoms except nearest neighbors interact through a Lennard-Jones potential. Four main atom types are distinguished: polar $(\mathrm{P})$, nonpolar $(\mathrm{N})$, apolar $(\mathrm{C})$, and charged $(\mathrm{Q})$. Nearest neighbors are connected by a weak harmonic spring, next nearest neighbors interact through a harmonic angle potential. Charged groups also interact through a short-range electrostatic potential. See text for details.

TABLE 1: Interaction Matrix ${ }^{a}$

\begin{tabular}{|c|c|c|c|c|c|c|c|c|c|c|c|}
\hline \multirow[b]{2}{*}{ group } & \multirow[b]{2}{*}{ subtype } & \multirow[t]{2}{*}{$\mathrm{P}$} & \multicolumn{4}{|c|}{$\mathrm{N}$} & \multirow[t]{2}{*}{$\mathrm{C}$} & \multicolumn{4}{|c|}{$\mathrm{Q}$} \\
\hline & & & 0 & $\mathrm{~d}$ & $\mathrm{a}$ & da & & 0 & $\mathrm{~d}$ & $\mathrm{a}$ & da \\
\hline $\mathrm{P}$ & & I & IV & III & III & II & V & I & I & I & I \\
\hline \multirow[t]{4}{*}{$\mathrm{N}$} & 0 & IV & III & III & III & III & III & III & III & III & III \\
\hline & d & III & III & II & II & II & IV & III & III & II & II \\
\hline & $\mathrm{a}$ & III & III & II & II & II & IV & III & II & III & II \\
\hline & $\mathrm{da}$ & II & III & II & II & I & V & III & II & II & I \\
\hline C & & V & III & IV & IV & V & III & V & V & V & V \\
\hline \multirow[t]{4}{*}{$\mathrm{Q}$} & 0 & I & III & III & III & III & V & III & III & III & II \\
\hline & d & I & III & III & II & II & V & III & III & II & I \\
\hline & $\mathrm{a}$ & I & III & II & III & II & V & III & II & III & I \\
\hline & da & I & III & II & II & I & V & II & I & I & I \\
\hline
\end{tabular}

${ }^{a}$ Level of interaction I (attractive), II (semiattractive), III (intermediate), IV (semirepulsive) or V (repulsive). Four different groups are considered: polar $(\mathrm{P})$, nonpolar $(\mathrm{N})$, apolar $(\mathrm{C})$, and charged $(\mathrm{Q})$. Both groups $\mathrm{N}$ and $\mathrm{Q}$ have four subtypes: 0 for no hydrogen bonding capabilities present, $\mathrm{d}$ for groups acting as hydrogen bond donor, a for groups acting as hydrogen bond acceptor, and da for groups with both donor and acceptor options.

Realistic masses can be assigned to the particles, but for reasons of computational efficiency, the same masses can be used. In the applications described here a mass of $m=72 \mathrm{amu}$ (corresponding to four water molecules) is assigned to each site unless otherwise stated. The mapping of some of the molecules studied in this paper is depicted graphically in Figure 1.

2.2. Nonbonded Interactions. The nonbonded interactions between interaction sites $i$ and $j$ are described by the LennardJones (LJ) potential

$$
U_{\mathrm{LJ}}(r)=4 \epsilon_{i j}\left[\left(\frac{\sigma_{i j}}{r}\right)^{12}-\left(\frac{\sigma_{i j}}{r}\right)^{6}\right]
$$

with $\sigma_{i j}$ representing the effective minimum distance of approach between two particles and $\epsilon_{i j}$ the strength of their interaction. The strength of the interaction, determined by the value of $\epsilon_{i j}$, is summarized in Table 1 for all possible interaction pairs. Note that only five levels of interaction are defined: attractive (I, $\epsilon$ $=5 \mathrm{~kJ} / \mathrm{mol}$ ), semiattractive (II, $\epsilon=4.2 \mathrm{~kJ} / \mathrm{mol}$ ), intermediate (III, $\epsilon=3.4 \mathrm{~kJ} / \mathrm{mol}$ ), semirepulsive (IV, $\epsilon=2.6 \mathrm{~kJ} / \mathrm{mol}$ ), and repulsive $(\mathrm{V}, \epsilon=1.8 \mathrm{~kJ} / \mathrm{mol})$. The level I interaction models strong polar interactions as in bulk water, level III models the nonpolar interactions in aliphatic chains, and level V models the hydrophobic repulsion between polar and nonpolar phases. 
Note that the level V interaction is actually still attractive but is labeled repulsive as it results in the demixing of water and oil phases. Levels II and IV are of intermediate strength. For all five interaction types the same effective size is assumed, $\sigma_{i j}$ $=0.47 \mathrm{~nm}$. In the simulations the LJ interaction potential is cutoff at a distance $r_{\text {cut }}=1.2 \mathrm{~nm}$, corresponding to approximately $2.5 \sigma$. To reduce the cutoff noise, the LJ potential is smoothly shifted to zero between a distance $r_{\text {shift }}=0.9 \mathrm{~nm}$ and $r_{\text {cut }}$. With the standard Gromacs shift function ${ }^{19}$ both the energy and the force vanish at the cutoff distance.

An alkane/water system was used as a reference system to calibrate the length and energy scales of the LJ parameters. A trial and error procedure was used to simultaneously optimize four basic parameters, namely the size of the particles plus the interaction energies between polar particles (level I), apolar particles (level III), and between polar and apolar particles (level V). Our goal was to reproduce the experimental densities of pure water and alkane systems around room temperature, the mutual solubility of oil and water, and the relative diffusion rates. Results are presented in the application section.

In addition to the LJ interaction, charged groups (type Q) interact via the normal electrostatic Coulombic potential

$$
U_{\mathrm{el}}(r)=\frac{q_{i} q_{j}}{4 \pi \epsilon_{0} \epsilon_{\mathrm{r}} r}
$$

with relative dielectric constant $\epsilon_{\mathrm{r}}=20$ for explicit screening. This potential is also shifted smoothly from $r_{\text {shift }}=0.0 \mathrm{~nm}$ to the same cutoff distance as used for the LJ interactions, $r_{\text {cut }}=$ $1.2 \mathrm{~nm}$. The standard shift function of Gromacs ${ }^{19}$ is used in which both the energy and force vanish at the cutoff distance. Shifting of the electrostatic potential in this manner mimics the effect of a distance-dependent screening. Note that interaction sites of type Q are intended for groups bearing full or close to full charges only. Interactions arising from small partial charges are represented by the LJ potential. The charges $q_{i}$ in principle represent the true charge of a group; however, in the case of small hydrated atoms (e.g., ions) a reduced charge is used to take into account the effect of an implicit hydration shell.

2.3. Bonded Interactions. Bonded interactions between chemically connected sites are described by a weak harmonic potential $V_{\text {bond }}(R)$ with an equilibrium distance $R_{\text {bond }}=\sigma=0.47$ nm:

$$
V_{\text {bond }}(R)=\frac{1}{2} K_{\text {bond }}\left(R-R_{\text {bond }}\right)^{2}
$$

The LJ interaction is excluded between bonded particles. Bonded particles, on average, are somewhat closer to each other than neighboring nonbonded particles (for which the equilibrium distance is $2^{1 / 6} \sigma$ ). The force constant of the harmonic bonding potential is $K_{\text {bond }}=1250 \mathrm{~kJ} \mathrm{~mol}^{-1} \mathrm{~nm}^{-2}$. This force constant allows considerable deviations from the equilibrium bond length $(\sim 15 \%)$ at the cost of $k T$. To represent chain stiffness, a weak harmonic potential $V_{\text {angle }}(\theta)$ of the cosine type is used for the angles:

$$
V_{\text {angle }}(\theta)=\frac{1}{2} K_{\text {angle }}\left\{\cos (\theta)-\cos \left(\theta_{0}\right)\right\}^{2}
$$

The basic equilibrium bond angle $\theta_{0}=180^{\circ}$, with a force constant of $K_{\text {angle }}=25 \mathrm{~kJ} \mathrm{~mol}^{-1} \mathrm{rad}^{-2}$. Such a small force constant allows an angle deviation of $30^{\circ}$ at the cost of $k T$. Note that this angle reproduces the properties of aliphatic chains. For angles of nonaliphatic nature comparison to atomistic models is used to optimize the parameters (see the various applications below). LJ interactions between second nearest neighbors are not excluded.

2.4. Simulation Parameters. The model is designed for use close to room or physiological temperatures. The temperature dependence of the model has not been investigated in detail. Standard coupling schemes can be used for both temperature and pressure. The largest feasible integration time step for most systems is $\mathrm{d} t=50 \mathrm{fs}$, but sometimes a smaller time step is required for stability $(30-40 \mathrm{fs})$. The neighbor list can be updated every 10 steps using a $1.2 \mathrm{~nm}$ neighbor list cutoff. Although the variables of the CG system (densities, length scales, energies, temperature, pressure) keep their physical meaning, this is not strictly true for the time scale. The dynamics are faster because the CG interactions are much smoother compared to atomistic interactions. On the basis of comparison of diffusion constants in the CG model and in atomistic models, the effective time sampled using CG is 3-6 times larger. Note that this factor affects ALL the dynamics present in the system. The relative dynamics present within the system appears to be well preserved (within a factor of 2). When interpreting the simulation results with the $\mathrm{CG}$ model, one can to a first approximation simply scale the time axis. The standard conversion factor we use is a factor of 4 , which is the effective speed up factor in the diffusional dynamics of $\mathrm{CG}$ water compared to real water. The simulation times reported in the remaining of the paper are effective times, unless otherwise stated.

The simulations were performed with the Gromacs simulation package version 3.0. ${ }^{19}$ For a typical system containing 100000 CG particles, a rate of 10000 time steps (2 ns effective time) per CPU hour on a dual Pentium $1 \mathrm{GHz}$ node is achieved. The parameters and example input files of the applications described in this paper are available at http://md.chem.rug.nl/ marrink/ coarsegrain.html.

\section{Applications}

3.1. Alkane/Water. The properties of bulk water can be reproduced by coarse graining four water molecules into one LJ bead of type P. At $T=300 \mathrm{~K}$ and $P=1$ bar a density of $\rho$ $=1 \mathrm{~g} \mathrm{~cm}^{-3}$ is obtained for a system containing $400 \mathrm{CG}$ particles (1600 real waters). From the volume fluctuations of this system the isothermal compressibility coefficient is found to be $6.0 \times$ $10^{-5} \mathrm{bar}^{-1}$, similar to the experimental value ${ }^{20}\left(4.5 \times 10^{-5}\right.$ bar $\left.^{-1}\right)$. The self-diffusion constant of the CG water sites at 300 $\mathrm{K}$ is $D_{\mathrm{CG}}=5 \times 10^{-6} \mathrm{~cm}^{2} \mathrm{~s}^{-1}$. The CG water sites, however, represent the center-of-mass of four real water molecules. The average mean squared displacement of the center-of-mass of four molecules is 4 times less than the average mean squared displacement of four independently diffusing molecules. ${ }^{15}$ The effective diffusion constant of individual water molecules as represented by the CG particles is therefore 4 times larger. The self-diffusion rate of water as modeled by the CG particles is therefore $2 \times 10^{-5} \mathrm{~cm}^{2} \mathrm{~s}^{-1}$. For pure water the experimental diffusion constant $D^{\exp }=2.3 \times 10^{-5} \mathrm{~cm}^{2} \mathrm{~s}^{-1}$ (at $300 \mathrm{~K}$ ).

To determine the freezing point of the $\mathrm{CG}$ water, a small ice cube was simulated surrounded by liquid water. At a temperature of $\simeq 290 \mathrm{~K}$ the solid phase appears in equilibrium with the liquid phase. Below this temperature the ice cube induces freezing of the whole system, and above this temperature the ice cube melts. The melting transition temperature of the CG water model is thus $T_{\text {melt }}=290 \pm 5 \mathrm{~K}$, slightly higher than for real water (273 $\mathrm{K})$. Spontaneous freezing of liquid water is only observed for temperatures below $240 \mathrm{~K}$, however. Within the temperature range of real liquid water the $\mathrm{CG}$ water therefore also remains 
TABLE 2: Properties of Water and Alkanes with the CG Model Compared to Experimental Values ${ }^{a}$

\begin{tabular}{llccc}
\hline \multicolumn{1}{c}{ system } & \multicolumn{1}{c}{ CG model } & $\begin{array}{c}\text { density, } \\
\mathrm{g} \mathrm{cm}^{-3}\end{array}$ & $\begin{array}{c}\text { compressibility, } \\
10^{-5} \text { bar }^{-1}\end{array}$ & $\begin{array}{c}\text { diffusion, } \\
10^{-5} \mathrm{~cm}^{2} \mathrm{~s}^{-1}\end{array}$ \\
\hline water & $\mathrm{P}$ & $0.99(0.99)$ & $6(4.5)$ & $2.0(2.3)$ \\
butane & $\mathrm{C}$ & $0.68(0.58)$ & $28(>17)$ & $1.9(>5)$ \\
hexane & $\mathrm{C}-\mathrm{C}$ & $0.58(0.66)$ & $14(17)$ & $0.7(4)$ \\
octane & $\mathrm{C}-\mathrm{C}$ & $0.77(0.70)$ & $14(13)$ & $0.6(2)$ \\
decane & $\mathrm{C}-\mathrm{C}-\mathrm{C}$ & $0.67(0.73)$ & $12(11)$ & $0.35(1)$ \\
dodecane & $\mathrm{C}-\mathrm{C}-\mathrm{C}$ & $0.80(0.75)$ & $12(10)$ & $0.3(-)$ \\
tetradecane & $\mathrm{C}-\mathrm{C}-\mathrm{C}-\mathrm{C}$ & $0.71(0.76)$ & $12(9)$ & $0.25(-)$ \\
hexadecane & $\mathrm{C}-\mathrm{C}-\mathrm{C}-\mathrm{C}$ & $0.81(0.77)$ & $12(9)$ & $0.2(-)$ \\
octadecane & $\mathrm{C}-\mathrm{C}-\mathrm{C}-\mathrm{C}-\mathrm{C}$ & $0.74(0.78)$ & $11(-)$ & $0.2(0.3)$ \\
eicosane & $\mathrm{C}-\mathrm{C}-\mathrm{C}-\mathrm{C}-\mathrm{C}$ & $0.82(0.79)$ & $11(-)$ & $0.15(-)$
\end{tabular}

${ }^{a}$ Properties at $300 \mathrm{~K}$, unless specified. Experimental properties are given in parentheses. ${ }^{b}$ Experimental densities at $293 \mathrm{~K}^{20}{ }^{2}$ Experimental isothermal compressibilities from ref 20. The values from simulations are computed from the volume fluctuations in an NPT ensemble. ${ }^{d}$ Diffusion rates were obtained from the slope of the mean squared displacement (MSD) curve in the long time limit. Experimental values extrapolated from temperature-dependent data. ${ }^{21}$

in a fluid state. Below a temperature of $\simeq 290 \mathrm{~K}$ the $\mathrm{CG}$ water is in a supercooled state.

Bulk properties of liquid alkanes can be reproduced using strings of connected $\mathrm{C}$ particles (each $\mathrm{C}$ particle representing four methyl/methylene groups) with standard bond and angle potentials (see above). We have performed bulk alkane simulations with our simplified force field for butane, octane, dodecane, hexadecane, and eicosane (1 through $5 \mathrm{C}$ particles, respectively). The systems contained 100 alkane molecules except for octane (200) and butane (400). The simulations were performed at 300 $\mathrm{K}$ and 1 bar using a Parrinello-Rahman coupling scheme. ${ }^{22}$ The densities, compressibilities, and self-diffusion rates obtained from the simulations are presented in Table 2. In this table we also present results for the intermediate alkanes such as hexane, decane, etc., which do not have a multiple of four methyl/ methylene groups. The properties of these molecules can best be approximated by assuming mapping less than four atomistic groups into a $\mathrm{CG}$ site; e.g., decane is modeled as $3 \mathrm{C}$ particles implying 3.3 to 1 rather than 4 to 1 mapping. Thus a simulation of $\mathrm{C}-\mathrm{C}-\mathrm{C}$ represents decane as well as dodecane. Only the masses assigned to each CG particle are different, resulting in different effective densities and diffusion rates. Especially for the longer alkanes (dodecane and up) the experimental densities are well reproduced (within 5\%) with the CG model. Pushing the limits of a coarse grained model, for the shorter alkanes the difference increases to $\sim 10 \%$. The experimental isothermal compressibilities are also approximated with the CG model over the whole range of chain lengths. The diffusion constants for the coarse grained alkane systems are obtained from the slope of the mean square displacement as a function of time, using the effective time scale set by the diffusion rate of pure water (see previous section). The self-diffusion of the alkanes appears 2-3 times slower than experimentally determined. Especially for the longer alkanes the agreement between the model and real alkanes is satisfactory for a CG model.

The chain stiffness of the CG alkanes is comparable to the chain stiffness of alkanes modeled atomistically. For comparison we performed atomistic simulations of alkane systems with the same number of molecules and simulation conditions as for the CG model. For these simulations we used the standard Gromacs force field, which is fully atomistic except for the methyl and methylene groups that are treated as unified atoms. In the CG model, the average $\mathrm{C}-\mathrm{C}-\mathrm{C}$ angle in a bulk phase of strings of three to five $\mathrm{C}$ sites is $140-142^{\circ}$ with a standard deviation of $20^{\circ}$ (only very weakly chain length dependent). Atomistic simulations of dodecane, hexadecane and eicosane give (136-

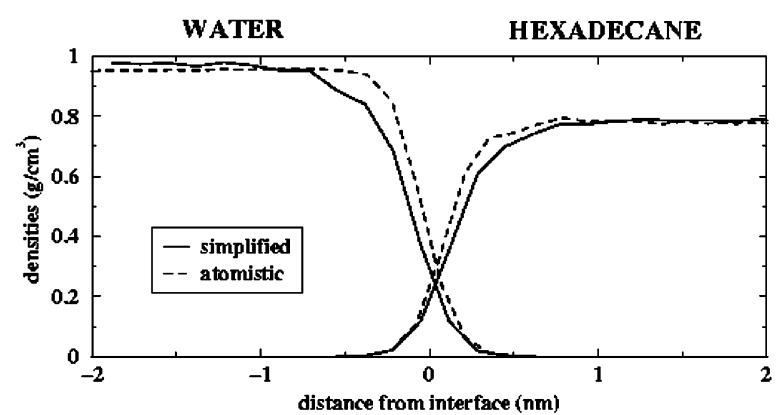

Figure 2. Hexadecane/water interface, from atomistic (dashed) and simplified (solid) simulations.

138) $\pm 26^{\circ}$, taking the center-of-mass of four adjacent methylene groups as the corresponding position of the $\mathrm{CG}$ site. A cis double bond can be effectively modeled by changing the equilibrium bond angle from $180^{\circ}$ to $120^{\circ}$. The chain stiffness is also increased slightly (from $K_{\text {angle }}=25$ to $35 \mathrm{~kJ} \mathrm{~mol}^{-1} \mathrm{rad}^{-2}$ ). In a simulation of strings of $5 \mathrm{C}$ particles in which the middle $\mathrm{C}-\mathrm{C}-\mathrm{C}$ angle is modeled as a double bond the average central angle is $122 \pm 15^{\circ}$. For comparison, in an atomistic simulation of octadecene the corresponding angle is $131 \pm 22^{\circ}$.

Apart from a correct representation of the relative densities of the oil and water phases, it is important in interface systems to reproduce oil/water mutual solubilities. To model the alkane/ water interface, randomly mixed systems containing either 100 hexadecane (C4) or 400 butane (C) molecules and 1600 water molecules (400 $\mathrm{P}$ particles) were prepared. The simulations were run for $1 \mu \mathrm{s}$ (CG, effective simulation time) or $5 \mathrm{~ns}$ (atomistic), at $T=300 \mathrm{~K}$ and an isotropic pressure of $P=1$ bar. All systems quickly phase separate to form an aqueous slab and an oil slab. In Figure 2 we compare the resulting relative densities of hexadecane and water obtained from simulations with simplified and with atomistic force fields. The density profiles are very similar. If the density within a CG site would be represented more realistically as smeared out over its entire volume, the matching is even better. The interfaces for the butane/water systems look very similar (not shown).

The solubility of water in oil can be computed straightforwardly from the equilibrium density $\rho_{\text {oil }}$ of water in the oil phase. The free energy of partitioning of water between the aqueous and the oil phase is given by $\Delta G=k T \ln \left(\rho_{\text {oil }} / \rho_{\text {wat }}\right)$, where $\rho_{\text {wat }}$ is the density of water in the aqueous phase. With the simplified model, simulations can be easily extended into the microsecond range, enough to obtain statistically reliable results on $\rho_{\text {oil }}$. The partitioning free energy of water in hexadecane for our simplified model was calculated to be $\Delta G=24 \pm 2 \mathrm{~kJ} \mathrm{~mol}^{-1}$. Experimentally, $\Delta G=25 \mathrm{~kJ} \mathrm{~mol}^{-1}{ }^{23}$ Likewise one could compute the solubility of hexadecane in water. The solubility of longer alkanes in water is so low $\left(\Delta G \simeq 50 \mathrm{~kJ} \mathrm{~mol}^{-1}\right)$, however, that even with the simplified model spontaneous partitioning into the aqeuous phase is not observed. Of course one could use other simulation methods to compute the free energy of such a process, but it was deemed more useful to look at the butane/water system. The experimental partitioning free energy for butane in water is $\Delta G=21 \mathrm{~kJ} \mathrm{~mol}^{-1} .^{24}$ From the observed equilibrium density of butane in water in our $\mathrm{CG}$ simulation, we estimate $\Delta G=22.5 \pm 2 \mathrm{~kJ} \mathrm{~mol}^{-1}$, also in good agreement with the experimental result. Both the solubility of water in alkane and of an alkane in water can be accurately reproduced in the $\mathrm{CG}$ force field.

3.2. Salt Solution. Pair distribution functions of sodium chloride at physiologically relevant concentrations $(0.1-1.0 \mathrm{M})$ can be approximated with $\mathrm{Q}$ particles bearing a reduced charge 


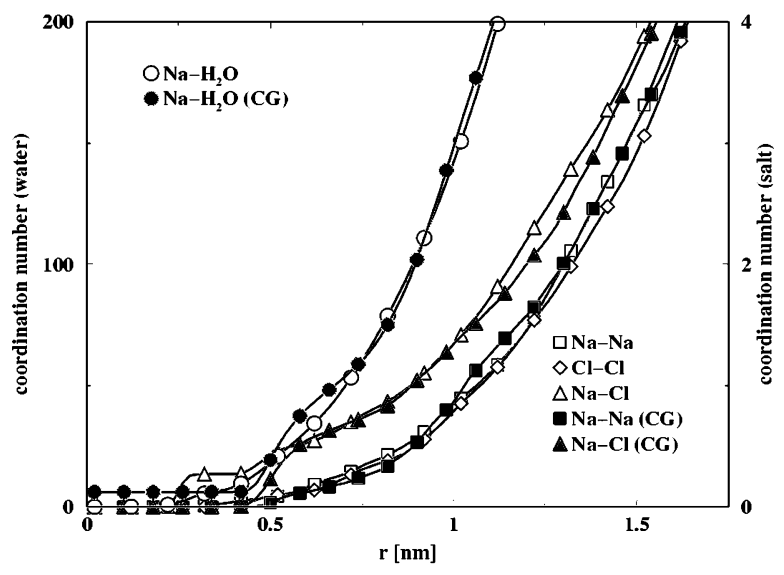

Figure 3. Cumulative pair distribution functions for a $0.4 \mathrm{M} \mathrm{NaCl}$ solution. Solid and open symbols denote data obtained with the CG model and atomistic model, respectively. Circles show sodium-water, triangles sodium-chloride, squares sodium-sodium, and diamonds chloride-chloride distribution functions. Note that within the CG model sodium and chloride are indistinguishable except for the opposite charge. $\mathrm{CG}$ waters are counted as four real water molecules. Six hydration waters are considered to be implicit in the CG ions.

of \pm 0.7 . The magnitude of this reduced charge (0.7) is merely a parameter, optimized to model the pair distribution functions obtained from atomistic simulations. In physical terms, the reduced charge mimics the implicit screening of the first hydration shell; i.e., the CG particle represents a hydrated ion. Experimentally, the first hydration shell of both sodium and chloride contains around six water molecules. ${ }^{25}$ Six hydration waters are therefore considered to be implicit in the CG ions. The implicit hydration shell implies strong hydrogen bonding possibilities. The ions are therefore modeled as subtype da. Given the short range nature of the electrostatic interaction in our model, the coarse grained ions are expected to model realistic ions in the limit of moderate to high ionic strength only (above $0.1 \mathrm{M}$ ). Although optimized for sodium chloride, the CG ions could be used for other salts as well.

In Figure 3 cumulative pair distribution functions obtained with the CG model are compared to those obtained from a reference atomistic simulation. The atomistic system contained 16 sodium chloride pairs and 2135 water molecules, a concentration of $0.4 \mathrm{M}$. A $10 \mathrm{~ns}$ simulation was performed at $T=300$ $\mathrm{K}$ using PME to account for the long-range electrostatic interactions. The CG system of the same solution consists of $32 \mathrm{Q}$ particles, 16 of which carry a positive charge of 0.7 and 16 a negative one of -0.7 . The number of solvent particles in the CG solution is 485 . Assuming four waters per CG water particle and six hydration waters implicit in the $\mathrm{Q}$ particle the concentration is also $0.4 \mathrm{M}$. The CG model was simulated for $1 \mu \mathrm{s}$. Figure 3 shows that the coordination numbers for both ion-ion and ion-water pairs obtained from atomistic simulations can be closely reproduced by the CG model for distances larger than $\sim 0.6 \mathrm{~nm}$. For smaller distances the atomistic nature of the particles becomes important which the CG model is unable to model. Similar agreement is found for ion concentrations in the range $0.1-1.0 \mathrm{M}$. Apparently, within this range, the electrostatic screening is sufficiently high that long-range interaction can be neglected.

3.3. Lipid Bilayers: DPPC. 3.3.1. CG Model. The phospholipid DPPC is modeled using $12 \mathrm{CG}$ sites (see Figure 1). The two tails (representing 15 methyl/methylene groups per tail) are based on the CG model for hexadecane. The glycerol ester linkage is modeled by two nonpolar particles, type $\mathrm{N}_{\mathrm{a}}$. The subtype a was chosen because of the hydrogen bond acceptor
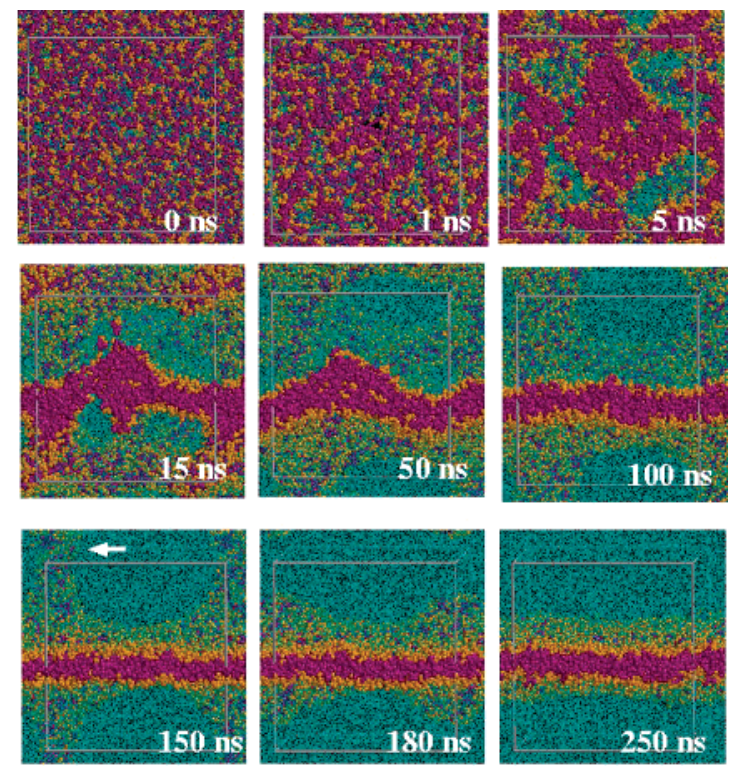

Figure 4. Spontaneous aggregation of DPPC lipids into a bilayer. Lipid headgroups are colored yellow, lipid tails purple, water blue. The simulation box is indicated with gray lines. The arrow points at a metastable lipid bridge (or elongated stalk).

capabilities of the carbonyl oxygens represented by these CG particles. The zwitterionic PC headgroup is modeled by a positively charged $\mathrm{Q}_{0}$ particle representing the choline moiety, and a negatively charged $\mathrm{Q}_{\mathrm{a}}$ particle representing the phosphate group. The possibility for the phosphate group to act as a hydrogen bond acceptor requires modeling as subtype a, whereas the choline group lacks hydrogen bonding options and is therefore modeled as subtype 0 . The angle potentials in the headgroup region were optimized to reproduce the values obtained in atomistic simulations. For the triplets GLYC-C1$\mathrm{C} 2$ and PO4-GLYC-C1 the same angle potentials are used as for the lipid tails, i.e., an equilibrium angle of $180^{\circ}$ and a force constant of $K_{\text {angle }}=25 \mathrm{~kJ} \mathrm{~mol}^{-1} \mathrm{rad}^{-2}$. An angle potential with a smaller equilibrium angle of $120^{\circ}$ was used to model the glycerol backbone PO4-GLYC-GLYC. The force constant was again $25 \mathrm{~kJ} \mathrm{~mol}^{-1} \mathrm{rad}^{-2}$.

3.3.2. Spontaneous Aggregation. Placed randomly into solution, the coarse grained DPPC molecules spontaneously form bilayers. Figure 4 shows an example of this aggregation process for a system containing 1600 DPPC molecules and $60000 \mathrm{CG}$ waters. A defect free bilayer is formed after $\sim 200 \mathrm{~ns}$ simulation. For systems containing fewer lipids the bilayer formation process is faster and comparable to the rate of formation observed for atomistic models. ${ }^{1}$ Systems with 128 lipids, for instance, self-assemble within tens of nanoseconds. The intermediate stages of bilayer formation are also similar to the stages found using atomistic models: a rapid local clustering of lipids into irregularly shaped aggregates on the subnanosecond time scale which coalesce to form a continuous lipid aggregate. This aggregate subsequently transforms itself into a bilayer configuration, still containing defects. Water pores appear metastable on time scales ranging between 3 and $25 \mathrm{~ns}$. In contrast to atomistically simulated bilayer assembly, with the CG approach another type of long living defect is observed, namely a lipid bridge (indicated by the arrow in Figure 4). A lipid bridge is an elongated stalk passing through the water phase connecting two bilayers, or more precisely the bilayer to its periodic image. It is the topological equivalent of a water pore that connects the water phases on both sides of the membrane running through the membrane. For small systems these lipid bridges are stable 


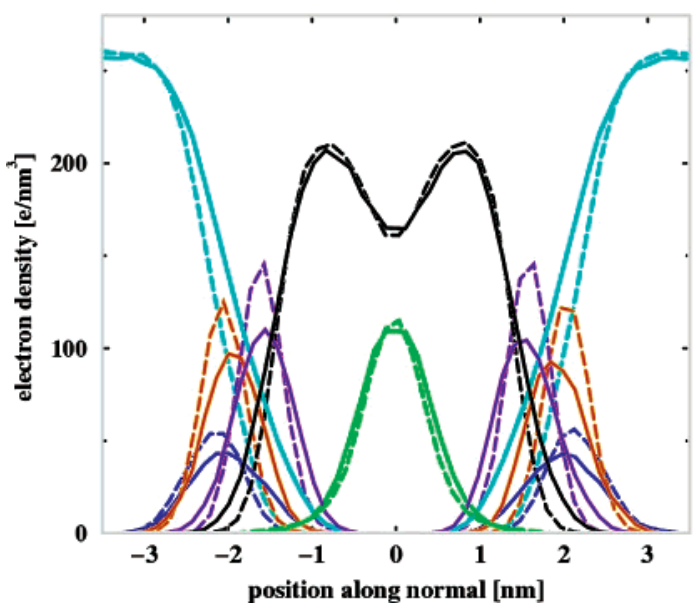

Figure 5. Comparison of electron density distributions obtained from atomistic (solid lines) and coarse grained (dashed) simulations. The water density is shown in cyan, the density of NC3 groups in blue, $\mathrm{PO}_{4}$ groups in orange, the glycerol backbone in purple, and the terminal tail groups in green. The total tail density is shown in black. The bilayer center is located at $0 \mathrm{~nm}$.

up to $20 \mathrm{~ns}$, typically slightly less stable than the water pores. For larger systems such as the one shown in Figure 4, however, it can take hundreds of nanoseconds before rupture occurs.

3.3.3. Structural Properties. Once these metastable defects have disappeared, the bilayer relaxes into its equilibrium structure. The equilibrium area per lipid for DPPC in the CG model at $323 \mathrm{~K}$ is $0.64 \mathrm{~nm}^{2}$. This matches the experimental estimate of $0.64 \mathrm{~nm}^{2} .^{26}$ Figure 5 compares the electron density profiles of an equilibrated coarse grained DPPC bilayer to profiles obtained for one simulated atomistically. Both density distributions were obtained from a simulation of a bilayer patch containing 256 DPPC lipids at full saturation (46 waters per lipid). The atomistic simulation was performed using a standard atomistic lipid force field ${ }^{27}$ and with PME for the long-range electrostatic interactions. To compare to the CG distributions, the center-of-mass of the atomistic groups corresponding to a CG particle were used to calculate the densities, except in the case of water for which this is not possible. Both the CG and atomistic simulations were performed at the same temperature $(T=323 \mathrm{~K})$ with the lateral and normal pressures independently coupled to a pressure of 1 bar to mimic conditions of zero surface tension. The pressure coupling scheme was ParrinelloRahman. ${ }^{22}$ The peaks in the electron density distributions coincide to within $0.1 \mathrm{~nm}$, for each of the membrane components. The water penetrates the bilayer to a larger extent in the atomistic simulations than in the CG simulations, but this is mainly a result of representing four real water molecules by the center of a single CG solvent particle. If the density were smeared out equally across the diameter of the CG particle (almost $0.5 \mathrm{~nm}$ ), the depth of water penetration becomes very similar in both cases. The interface in the CG model is slightly more structured than in the atomistic model. The thickness of the CG bilayer, measured from the peaks of the phosphate distribution, measures $4.0 \pm 0.1 \mathrm{~nm}$, close to the experimentally determined bilayer thickness of $3.85 \mathrm{~nm}$ for the lamellar phase of DPPC in the liquid-crystalline phase. ${ }^{26}$ The close correspondence between the structure of the CG bilayer and the atomistic one is further illustrated in Figure 6, which shows an alignment of snapshots of both systems.

For further comparison, in Figure 7 lipid order parameters are shown for both the CG and the atomistic DPPC bilayer. The second-rank order parameter $P_{2}=1 / 2\left(3 \cos ^{2} \theta-1\right)$ was computed for consecutive bonds, with $\theta$ the angle between the
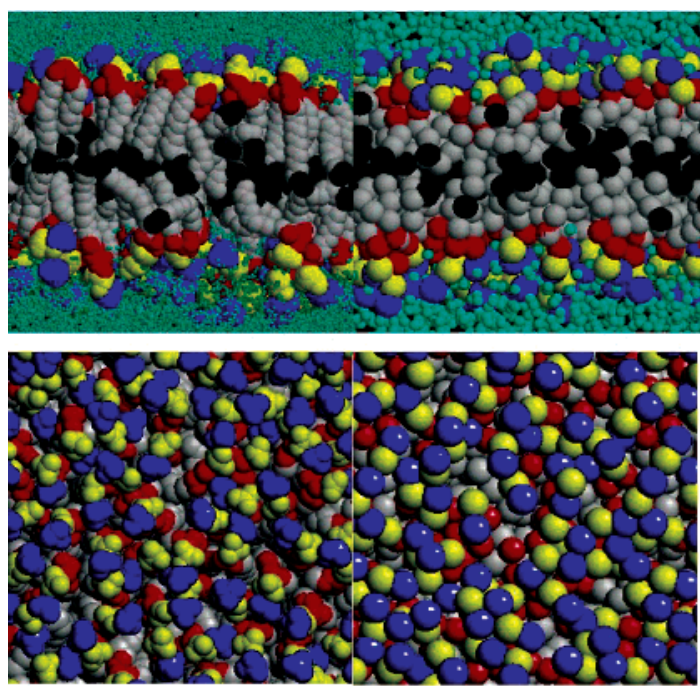

Figure 6. Snapshots of a DPPC bilayer simulated with an atomistic model (left) and with the CG model (right). The top images show side views of the bilayer, the bottom images show top views (with the water omitted). The choline groups are colored blue, phosphatidyl groups yellow, the glycerol backbone red, the tails gray. A darker shade of gray is used to distinguish the terminal methyl group. Water is colored cyan.

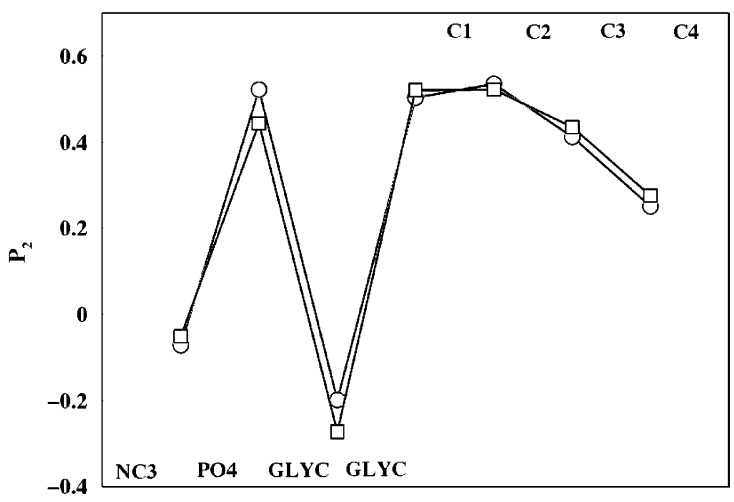

Figure 7. $P_{2}$ order parameter of consecutive bonds with respect to the bilayer normal. Results obtained for the CG bilayer are marked with circles, those for the atomistic bilayer with squares. Data are averaged over both tails.

direction of the bond and the bilayer normal. Perfect alignment with the bilayer normal is indicated by $P_{2}=1$, perfect antialignment with $P_{2}=-0.5$, and a random orientation with $P_{2}$ $=0$. For the atomistic simulation the center-of-mass of four adjacent methylene groups was taken as the position corresponding to the position of a CG site. The profiles are very similar. Both the phosphate-choline bond and the glycerol linkage have a predominantly parallel orientation with respect to the surface normal, whereas the other bonds prefer aligning along with the surface normal. Toward the end of the tails, the order decreases. Note that the order parameters obtained here cannot be directly compared to experimental bond order parameters, as the information about atomistic bonds is not present in the CG model. However, good agreement between experimental and atomistically simulated order parameter profiles have been shown in the past. ${ }^{27}$

3.3.4. Elastic Properties. Apart from the ability to reproduce important structural quantities, collective elastic properties such as the bilayer bending modulus, the area compressibility, and the line tension are important parameters to judge the quality of the model. The bending modulus can be obtained from the undulation spectrum of a large patch of bilayer, as described in 


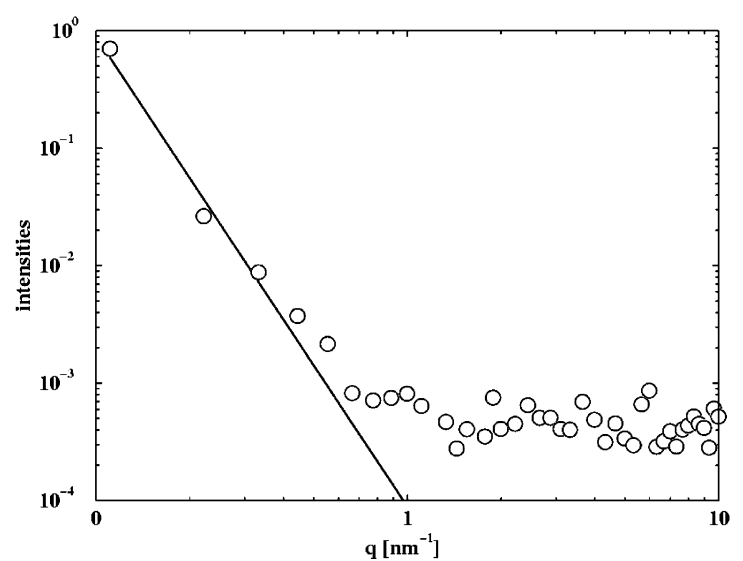

Figure 8. Spectral intensity for undulatory modes as a function of wavenumber $q$. The solid line represents a fit to the data at long wavelengths, obeying $q^{4}$ behavior.

ref 28. For this purpose the small bilayer containing 256 lipids was copied five times in both lateral directions to generate a patch consisting of 6400 lipids with lateral dimension $\sim 45 \mathrm{~nm}$. The system was simulated for $250 \mathrm{~ns}$ at the same conditions as described above. It took $100 \mathrm{~ns}$ for the undulatory modes to fully develop. Figure 8 shows the undulatory spectrum obtained from the final $150 \mathrm{~ns}$ simulation of this system. The intensity $I$ $=\left\langle u^{2}\right\rangle$ of the undulations with amplitude $u$ obey $q^{4}$ behavior in the long wavelength regime, as predicted by a continuum model, ${ }^{29}$ i.e., $I \propto k T\left(A k_{\mathrm{c}} q^{4}\right)^{-1}$. Here $A$ is the membrane area and $k_{\mathrm{c}}$ denotes the bending modulus. Fitting this equation to the data obtained from the simulation results in a bending modulus of $4 \pm 2 \times 10^{-20} \mathrm{~J}$, where the uncertainties are determined from different fitting procedures. This value is close to the value of $5 \times 10^{-20} \mathrm{~J}$ obtained for an atomistically simulated DPPC bilayer ${ }^{27}$ and also to the value of $(5.6 \pm 0.6)$ $\times 10^{-20} \mathrm{~J}$ obtained experimentally for DMPC. ${ }^{30}$

The area compressibility modulus $K_{\mathrm{A}}$ can be calculated from the fluctuations in the membrane area per lipid $A: K_{\mathrm{A}}=$ $k T\langle A\rangle\left(N\left\langle\left(A-A_{0}\right)^{2}\right\rangle\right)^{-1}$, where $N$ denotes the number of lipids per monolayer and $A_{0}$ denotes the equilibrium area. For the large bilayer patch containing 6400 lipids the area compressibility modulus is found to be $K_{\mathrm{A}}=260 \pm 40 \mathrm{mN} / \mathrm{m}$. For the smaller system consisting of 256 lipids the value is higher, $K_{\mathrm{A}}=400$ $\pm 30 \mathrm{mN} / \mathrm{m}$. The difference is due to the contribution of undulatory modes in the large system which lower the area compressiblity. ${ }^{28}$ The value obtained for the large system compares well with the experimental value for DPPC which is reported to be $K_{\mathrm{A}}=231 \pm 20 \mathrm{mN} / \mathrm{m} .{ }^{26}$ It is reasonable to assume that the experimental value also contains contributions from undulatory modes. Note that atomistic simulations performed on small bilayer patches usually also report $K_{\mathrm{A}}$ values that are a factor two larger than the experimental estimate (e.g., see ref 31).

The line tension, or edge energy, of the lipid membrane can be obtained from the critical tension at which pores can be stabilized inside a membrane. According to a theoretical model ${ }^{32}$ the energy $E$ of a pore inside a membrane is given by the expression $E(r)=2 \pi \lambda-\pi r^{2} \gamma$, where $r$ denotes the pore radius, $\lambda$ the line tension, and $\gamma$ the surface tension. At low tension the pores are unstable. The edge energy drives the closure of the pore. However, at a critical tension $\gamma^{*}$ the edge energy is overcome. Pores can then be stabilized at $r=\gamma^{*} / \lambda$. For larger tensions pore growth is unlimited, leading to membrane rupture. The tension-dependent opening and closing of a pore in the membrane has recently been simulated for DPPC using an

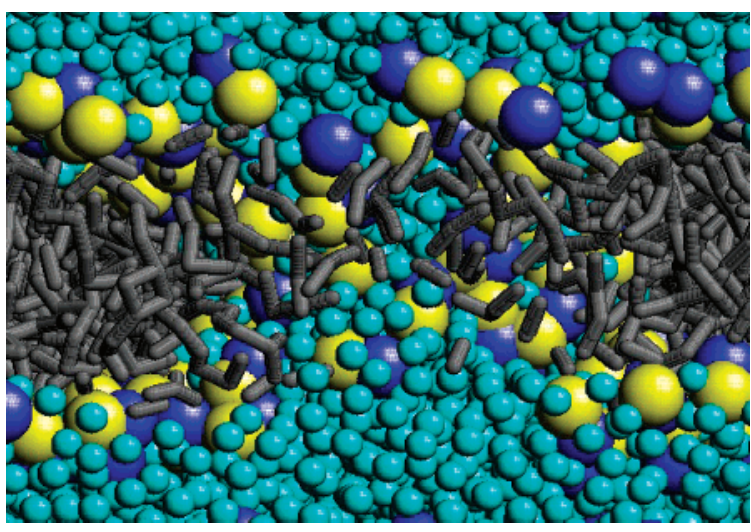

Figure 9. Close up of a pore stabilized in a coarse grained DPPC membrane at a surface tension of $\gamma \sim 25 \mathrm{mN} / \mathrm{m}$.

atomistic model. ${ }^{33}$ It was found that pores of radius $r=0.9 \mathrm{~nm}$ appeared stable at a critical tension of $\gamma^{*} \sim 20-40 \mathrm{mN} / \mathrm{m}$ (depending on details of the force field), corresponding to a line tension of $\lambda=(1-3) \times 10^{-11} N$. This value is of the same order of magnitude as the estimates from experiments using electroporation techniques $\lambda=1 \times 10^{-11} N^{34}$ and $\lambda=2 \times$ $10^{-11} N$ for natural lecithins and $\lambda=1 \times 10^{-11} N^{35}$ for SOPC, and from mechanical rupture events $\lambda=1 \times 10^{-11} N^{36}$ for DOPC and SOPC. Repeating the simulations as described in ref 33, using the CG force field, quantitatively similar results are found. Large tensions are required to spontaneously induce pore formation, immediately followed by membrane rupture. At a tension of $100 \mathrm{mN} / \mathrm{m}$ a pore appears within nanoseconds; at a tension of $65 \mathrm{mN} / \mathrm{m}$ it takes several microseconds. At smaller tensions $(<50 \mathrm{mN} / \mathrm{m})$ the membrane thins, but no pore formation is observed on time scales up to $50 \mu \mathrm{s}$. The thinning of the membrane at low tensions $(5-10 \mathrm{mN} / \mathrm{m})$ is comparable to that observed experimentally $(1-2 \%) .{ }^{37}$ Preexisting pores in the membrane can be stabilized at a critical tension of $\gamma \sim 25$ $\mathrm{mN} / \mathrm{m}$. The pores are similar in shape to those reported for the atomistic simulations, ${ }^{33}$ i.e., an hourglass shaped water channel lined by lipid headgroups. Figure 9 shows a closeup of such a pore stabilized in a coarse grained DPPC membrane. The radius of the pore fluctuates between 1.5 and $2.0 \mathrm{~nm}$, from which the line tension is estimated to be $\lambda=(5 \pm 1) \times 10^{-11} N$. This value for the line tension is slightly higher, but of the same order of magnitude, as the experimental values cited above.

3.3.5. Dynamical Properties. As discussed above, the rate of spontaneous aggregation of lipids into bilayers using the CG model is similar to the rates found for atomistic simulations. The lateral diffusion constant of the lipids can be obtained from the limiting slope of the MSD curve. Microsecond simulations of the bilayer containing 256 DPPC molecules allows the observation of truely long time diffusive behavior. At $T=323$ $\mathrm{K}$, the lateral diffusion rates of DPPC equals $(3 \pm 1) \times 10^{-7}$ $\mathrm{cm}^{2} \mathrm{~s}^{-1}$, of the same order of magnitude as experimentally measured (values are typically reported to be around $1 \times 10^{-7}$ $\mathrm{cm}^{2} \mathrm{~s}^{-1}$ at temperatures close to $323 \mathrm{~K}$; e.g., see refs 38 and 39).

Another interesting dynamic quantity for which experimental data exist, is the rate of permeation of water across the bilayer. During microsecond simulations of a 256 DPPC bilayer patch the spontaneous permeation of water molecules is observed. The magnitude of the unidirectional flux $J$ is $1 \mathrm{CG}$ water molecule per $100 \mathrm{~ns}$, corresponding to 4 real waters. From this flux the permeation rate can be computed as $P=J / \triangle C A=(1.5 \pm 0.5)$ $\times 10^{-3} \mathrm{~cm} \mathrm{~s}^{-1}$, where $\Delta C=55.5 \mathrm{~mol} \mathrm{dm}^{-3}$ is the effective water concentration and $A$ denotes the membrane area $(A=81$ 


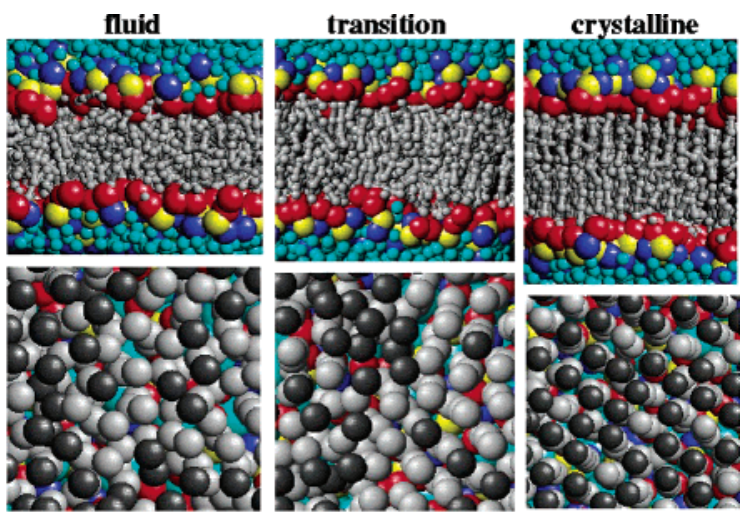

Figure 10. Thermal phase transition from liquid-crystalline to crystal phase. A DPPC bilayer patch in the fluid phase (left) was cooled to $283 \mathrm{~K}$. After $20 \mathrm{~ns}$ the system underwent a cooperative phase transition (middle) and relaxed into a crystal phase (right).

$\mathrm{nm}^{2}$ ). The magnitude of the permeation rate is of the same order of magnitude as the experimentally measured permeability coefficients for pure DPPC vesicles. ${ }^{40,41}$

3.3.6. Crystalline Phase. Upon cooling of the 256 DPPC bilayer patch below a temperature of $283 \mathrm{~K}$, formation of a crystalline phase can be observed. In Figure 10 this process is shown in a series of snapshots. At $T=283 \mathrm{~K}$ the bilayer remains in a fluid phase for tens of nanoseconds, before the transition takes place. The transition is observed to be highly cooperative, taking place within a few nanoseconds. The area per headgroup in the CG crystalline phase is $0.47 \mathrm{~nm}^{2}$, with the tails packed in a hexagonal lattice. Some packing defects exist, however, as can be seen from the snapshots. The absence of lipid lateral diffusive motion implies a crystalline state rather than a gel phase. The lipid headgroups remain mobile. Experimentally, ${ }^{42}$ DPPC forms a crystalline phase $\left(\mathrm{L}_{\mathrm{c}}\right)$ below temperatures of $\sim 273 \mathrm{~K}$ with an area per headgroup of $0.46 \mathrm{~nm}^{2}$. In the experiment the DPPC tails are tilted with respect to the bilayer normal with a tilt angle close to $35^{\circ}$. Although the simulation box is fully adjustable allowing any preferential packing, no spontaneous tilt is observed. Upon heating of the system, melting is observed at temperatures above $300 \mathrm{~K}$. Whether a gel phase can also be stabilized as an intermediate phase is not yet clear.

3.4. Lipid Bilayers: Other Phospholipids. Phospholipids other than DPPC can also be readilly modeled using the CG approach. The tail length can be modified by removing or adding additional apolar sites. On the basis of the optimal mapping for alkanes (see section 3.1), a lauroyl tail is modeled with $3 \mathrm{C}$ sites, both miristoyl and palmitoyl with 4 , and stearoyl with 5 . Cis double bonds can be modeled in a similar manner as in alkenes (see section on alkane/water) by setting the equilibrium angle of the CG triplet, which includes the double bond to $120^{\circ}$, and increasing the chain stiffness slightly to $K_{\text {angle }}=35 \mathrm{~kJ} \mathrm{~mol}^{-1}$ $\mathrm{rad}^{-2}$. A phosphatidylethanolamine (PE) headgroup can be modeled by two $Q$ particles just as for PC. To mimic the strong hydrogen bond donor capabilities and indirect acceptor capabilities (via water meditated hydrogen bonds) of the ethanolamine site compared to the choline site, the $Q$ particle representing the ethanolamine group is of the more polar subtype da.

The area per headgroup obtained from CG simulations for a number of common phospholipids is reported in Table 3 . The simulations were performed on patches of 256 lipids with a hydration level of 40 waters/lipid, close to, or beyond, the swelling limit of most phospholipids. The simulations cover a range of physiologically relevant temperatures. Zero surface tension conditions apply to all systems. Given the uncertainty in experimental measurements, ${ }^{26}$ the CG model performs well,
TABLE 3: Area per Lipid for Common Phospholipids in the CG Model

\begin{tabular}{llll}
\hline system & $\begin{array}{c}\text { simulated area, } \\
\mathrm{nm}^{2}\end{array}$ & \multicolumn{1}{c}{ experimental area, ${ }^{a} \mathrm{~nm}^{2}$} & \multicolumn{1}{c}{ ref } \\
\hline DPPC & $0.47^{b}(283 \mathrm{~K})$ & $0.46^{b}(273 \mathrm{~K}), 0.48^{c}(293 \mathrm{~K})$ & 42,26 \\
DPPC & $0.59(300 \mathrm{~K})$ & & \\
DPPC & $0.64(323 \mathrm{~K})$ & $0.64(323 \mathrm{~K})$ & 26 \\
DPPC & $0.66(338 \mathrm{~K})$ & $0.64-0.67^{d}(333 \mathrm{~K}), 0.67(338 \mathrm{~K})$ & 43,44 \\
DLPC & $0.60(300 \mathrm{~K})$ & $0.57(293 \mathrm{~K}), 0.63(303 \mathrm{~K})$ & 43,44 \\
DLPC & $0.66(338 \mathrm{~K})$ & $0.64-0.68^{d}(333 \mathrm{~K}), 0.71(338 \mathrm{~K})$ & 43,44 \\
DSPC & $0.66(338 \mathrm{~K})$ & $0.65(333 \mathrm{~K}), 0.66(338 \mathrm{~K})$ & 43,44 \\
DOPC & $0.67(300 \mathrm{~K})$ & $0.72(303 \mathrm{~K})$ & 26 \\
DPPE & $0.62(338 \mathrm{~K})$ & $0.61(342 \mathrm{~K})$ & 44 \\
DOPE & $0.61(273 \mathrm{~K})$ & $0.65(271 \mathrm{~K})$ & 45 \\
DLPE & $0.55(300 \mathrm{~K})$ & $0.51(308 \mathrm{~K})$ & 26 \\
POPE & $0.59(300 \mathrm{~K})$ & $0.57(303 \mathrm{~K})$ & 45
\end{tabular}

${ }^{a}$ Simulation results are accurate to within $0.01 \mathrm{~nm}^{2}$. Experimental results are subject to substantial interpretation uncertainties (typically not known with a precision of more than $\left.0.02 \mathrm{~nm}^{2}\right) .{ }^{26}{ }^{b}$ Crystalline phase. ${ }^{c}$ Gel phase. ${ }^{d}$ Extrapolated estimates.

at the same level of accuracy as atomistic force fields. Crucial effects such as the condensation of the PE headgroup with respect to the PC headgroup (compare DPPC/DPPE at $338 \mathrm{~K}$ ), the area expansion upon temperature increase (DPPC at 300$323-338 \mathrm{~K}$ ) or upon chain unsaturation (e.g., DOPC/DPPC at $300 \mathrm{~K})$, as well as the negligible effect of chain elongation (DLPC-DPPC-DSPC at $338 \mathrm{~K}$ ) are all reproduced.

3.5. Nonlamellar Phases. In this section we show that the coarse grained model can also be applied to study nonlamellar phases. Although optimized and tested for bilayer systems, there is no bias in the model favoring lamellar phases. This section describes two successful applications in which lipids spontaneously aggregate into either an inverted hexagonal phase or a micellar phase in agreement with the experimentally determined phase.

3.5.1. Inverted Hexagonal. Dioleoylphosphatidylethanolamine (DOPE) experimentally forms an inverted hexagonal phase at temperatures above $\sim 280-300 \mathrm{~K}$ (depending on hydration conditions). ${ }^{46}$ The maximum amount of water that can be taken up by the hexagonal phase is about 20 waters per lipid. To study the phase preference of the CG model for DOPE (see previous section), a system consisting of 1024 DOPE lipids randomly solvated into 4224 solvent sites (16 real waters/lipid) was generated. The pressure scaling was performed completely anisotropically, allowing the deformation of the box shape and the development of hexagonal symmetry. The system was simulated at two different temperatures: 273 and $318 \mathrm{~K}$. In agreement with the experimental behavior, at the lower temperature DOPE aggregates into a lamellar phase, whereas at the higher temperature an inverted hexagonal phase forms spontaneously. Repeated simulations with different random starting conditions show the same behavior. The aggregation into the inverted hexagonal phase can be divided into four substages: (i) rapid clustering of water into a network of connected inverted micelles, (ii) disappearance of some and growing of other aqeuous connections leading to the formation of two inverted cylindrical micelles, (iii) connection of these micelles with their periodic image resulting in the formation of two water channels, and (iv) relaxation of the water channels into a perfect hexagonal lattice. The first stage is complete within a few nanoseconds. Stages two and three, which are not always well distinguished, together take $\sim 100 \mathrm{~ns}$. The final relaxation is accomplished within tens of nanoseconds. As with the formation of bilayers (see the section on DPPC), the dynamics of self-aggregation is very dependent on system size. Spontaneous aggregation runs for systems 8 times larger remain trapped 


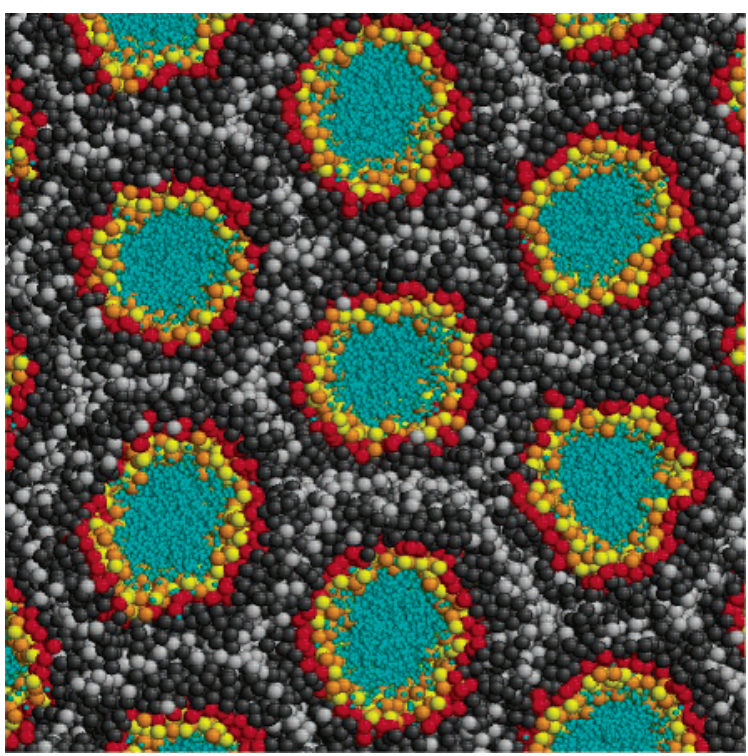

Figure 11. Snapshot of an inverted hexagonal phase of DOPE at 318 $\mathrm{K}$. The system contains more than 8000 lipids and at 16 waters/lipid. The ethanolamine groups are colored orange, phosphatidyl groups yellow, the glycerol backbone red, the tails gray. A lighter shade of gray is used to distinguish the terminal methyl group. Water is colored cyan. The system is viewed along the direction of the water channels. Note the hexagonal structure of the channels themselves.

at substage two (with many inverted cylindrical micelles) at least up to the microsecond time scale. A simulation of a system consisting of eight copies of the relaxed inverted hexagonal phase (more than 8000 lipids) was stable, however. The final snapshot (at $0.5 \mu \mathrm{s}$ ) of this system, with eight independent water channels, is shown in Figure 11. The water channels are clearly arranged in a hexagonal pattern. The lipids have adopted an inverted geometry with the headgroups confined into the small area around the channels, leaving ample space for the tails that resist tight packing due to the presence of a double bond in each tail. Remarkably, the geometry adapted by the water channels is not spherical, but hexagonal. Experimentally the details of the packing are not known, but both a spherical and a hexagonal arrangement have been proposed. ${ }^{46,47}$ The hexagonal packing around the channels also shows up at the border between the two monolayer leaflets, reflected by the distribution of the terminal tail group. The hexagonal spacing in the simulations is $5.7 \mathrm{~nm}$, which is in agreement with the experimental spacing (estimated to be $5.7-5.8 \mathrm{~nm}$ at $T=318 \mathrm{~K}$ with 16 waters/lipid) determined from X-ray analysis of the inverted hexagonal phase of DOPE. ${ }^{46}$ A more extensive analysis of the structure of simulated inverted hexagonal phases and a more elaborate comparison to the available literature data will be published separately.

3.5.2. Micelle. Removing the glycerol linkage as well as one of the tails, and one site of the remaining tail from DPPC, the five remaining sites model dodecylphosphocholine (DPC). The mapping of the DPC lipid in the CG model is shown in Figure 1. Experimentally, DPC forms micelles at concentrations above the critical micelle concentration $(\mathrm{cmc} \sim 1 \mathrm{mM}) .{ }^{48}$ A system composed of 400 DPC lipids randomly solvated by 125,000 $\mathrm{CG}$ waters was simulated for a microsecond. The concentration of lipids is $0.04 \mathrm{M}$, well above the cmc. The DPC lipids indeed spontaneously form small micelles. The micellar size distribution does not converge within a microsecond, however. Encounters between micelles are diffusion-limited and therefore rare. The exchange rate of lipids from micelles into solution is also too slow to allow for a rapid equilibration. The final distribution is

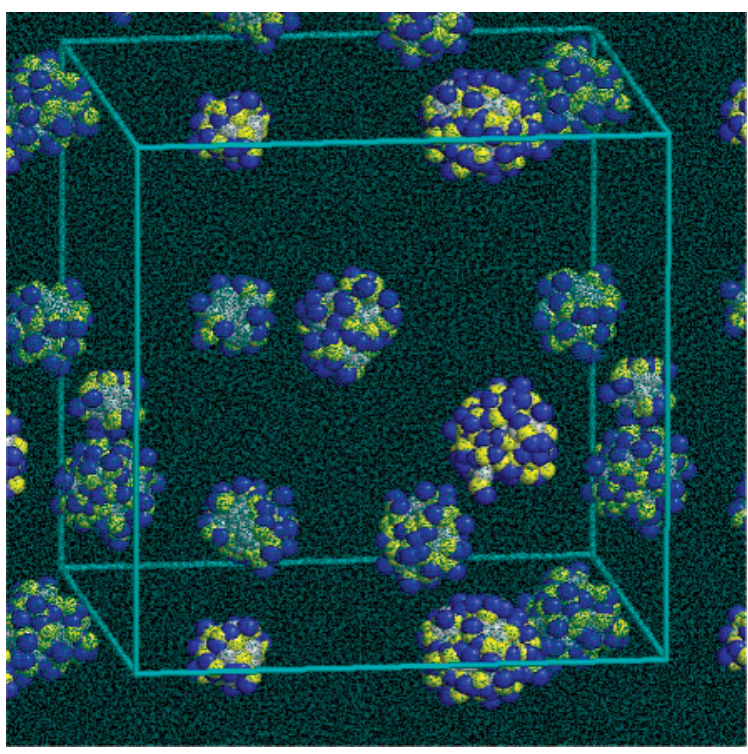

Figure 12. Distribution of coarse grained DPC micelles after $1 \mu$ s of simulation. The choline groups are depicted blue, the phosphate yellow, and the tails gray. Water is colored cyan. One periodic image of the simulation box is also depicted. The box edge length is $25 \mathrm{~nm}$.

shown in Figure 12. A few smaller DPC clusters $(<20)$ still exist, but most lipids are organized in small spherical micelles. The size of these micelles ranges from 40 to 70 . Experimentally the size of DPC micelles is determined as $56 \pm 5$ (at $0.02 \mathrm{M}^{48}$ ).

Aggregation of a smaller system consisting of 54 DPC lipids and $5600 \mathrm{CG}$ waters $(0.12 \mathrm{M})$ was also studied to compare the rate of aggregation to results obtained with atomistic simulations for a similar system. ${ }^{49}$ The initial rate of clustering of single lipids into small clusters of a few lipids takes place within a nanosecond for both the CG and the atomistic system. Subsequent formation of larger clusters and micelles, however, is clearly faster in the atomistic simulations. The formation time of the final micelle (containing all 54 lipids) in the atomistic simulation was $12 \mathrm{~ns}$, whereas with the CG model it takes between 50 and $150 \mathrm{~ns}$ in four independent attempts. The reason for the different dynamics is likely the absense of long-range attraction in the CG model. The radial structure of the final CG micelle is very similar to that of the atomistic one. In Figure 13 we compare the radial atom density distribution of the $\mathrm{CG}$ micelle to an atomistic one simulated by Tieleman et al., ${ }^{50}$ also containing 54 DPC molecules. The distribution of the various atom groups inside the micelle is very similar. Only the dip in density in the center of the micelle appears more pronounced in the atomistic simulation. The micelle radius is $\sim 2 \mathrm{~nm}$ (to the phosphate peak) in both systems.

\section{Discussion}

In the introduction four cornerstones for a successful coarse grained model were defined: speed, accuracy, applicability, and versatility. The coarse grained model presented in this paper has been optimized to match these criteria as well as possible. (i) Speed: A speed up factor between 10 and 25 is obtained due to the use of a $50 \mathrm{fs}$ time step instead of 2-5 fs maximum for atomistic simulations. The reduced number of interaction sites allows simulations approximately between 5 (for force field with unified methylene groups) and 10 times faster (for force fields that have explicit hydrogens). Furthermore, the short-range nature of the interactions speeds up the calculations between 3 times (compared to simulations using typical cutoffs of $1.4 \mathrm{~nm}$ ) and 10 times (when Ewald summation type techniques are used 


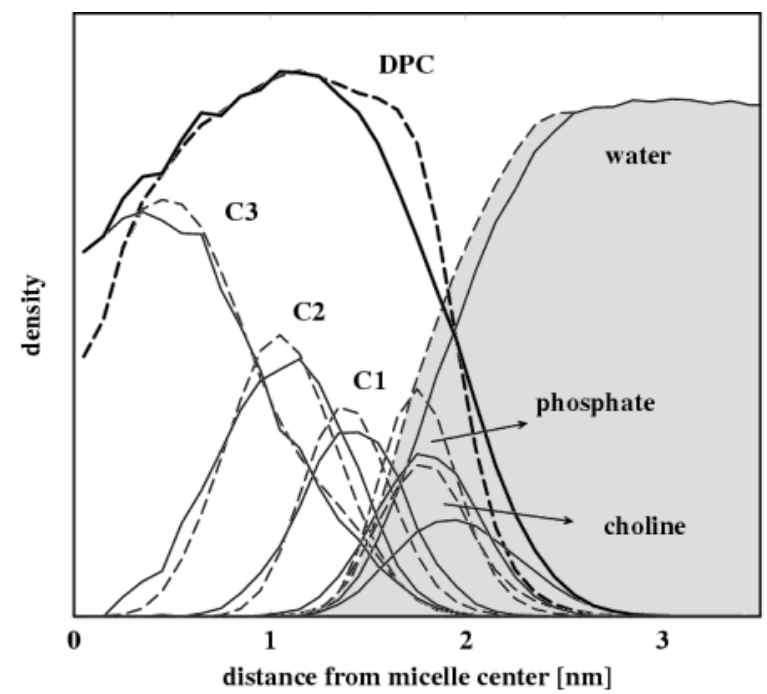

Figure 13. Radial distribution functions of a DPC micelle, from atomistic simulations (dashed lines) and CG simulations (solid lines).

to treat the long-range interactions). The increased dynamics in the CG model results in another effective speed up with a factor of $\sim 4$. Together, these factors result in a speed up of 3-4 orders of magnitude with the CG model compared to currently used atomistic simulation techniques. Also compared to the semiquantitative CG model of Shelley et al. ${ }^{18}$ the current model is much faster and comparable to the speed obtained with DPD techniques. (ii) Accuracy: The CG model is shown to be accurate at least at a semiquantitative level for structural, elastic, dynamic as well as thermodynamic properties for a range of lipid systems. Structural properties of lipid systems such as the area per headgroup in the lamellar phase or the hexagonal spacing in the inverted hexagonal phase agree well with the available experimental data. Compared to results obtained with atomistic simulations, atom density distributions are very similar in all cases considered. Elastic properties computed for a DPPC bilayer such as the bending modulus, the line tension or the area compressibility are of the same order as the experimental measurements. The absolute dynamics in the CG model is faster compared to real systems or simulations with atomistic force fields. The relative dynamics, however, appears well preserved. With a time conversion factor of 4 a variety of dynamic properties such as self-diffusion in bulk phases and lipid lateral diffusion, the permeation rate of water across a membrane, or the lipid self-aggregation rate are meaningful at a semiquantitative level. Thermodynamically speaking, the CG model has encouraging properties too. Not only is the mutual solubility of water and alkane well reproduced, but more importantly lipids aggregate into the correct phases whether lamellar, micellar or hexagonal. For DPPC a freezing transition was even observed when the temperature was lowered below the main phase transition temperature. (iii) Applicability: The use of physically meaningful parameters makes the model easy to interpret. Instead of dealing with reduced units that need conversion afterward, it is immediately clear what the state conditions are. The limited number of particle types and interaction levels provide for a small set of building blocks from which related molecules that are expected to perform at the same level of accuracy as the examples given in this paper can be easily constructed. (iv) Versatility: Although optimized mainly for lipid systems in the lamellar phase, the CG model has no built in restrictions as to the phase of the system. The lipids in the CG model are very flexible, free to adapt (almost) any conformation at a reasonable energy cost. Two applications to nonlamellar systems were used to illustrate this versatility. Recently, the CG model has also been successfully applied to study the spontaneous formation of vesicles, ${ }^{51}$ and to the fusion mechanism of vesicles. ${ }^{52}$ Apart from phospholipid systems, the CG model can in principle be applied to other types of molecules as well. For instance, currently a CG topology is being developed for cholesterol.

Of course the CG model as presented in this paper is limited. There will be many applications for which it is not well suited. Further optimizations are possible but as with any CG model its utility is inherent in its simplicity. Any application for which long-range electrostatic forces are important should be considered with care. Fine chemical detail is inaccessible in any coarse grained approach. One should be careful not to overinterpret the results on a quantitative level. The CG model is not a tool to replace atomistic simulations, but rather the two should be used side by side. With the CG model the long time-scale or length-scale properties of the system of interest can be explored, whereas with atomistic models the details can be studied. Results form atomistic simulations should be used as much as possible to judge the quality of the CG force field in the application at hand.

\section{References and Notes}

(1) Marrink, S. J.; Lindahl, E.; Edholm, O.; Mark, A. E. J. Am. Chem. Soc. 2001, 123, 8638 .

(2) Shelley, J. C.; Shelley, M. Curr. Opin. Colloid Interface Sc. 2000, 5,101 .

(3) Müller, M.; Katsov, K.; Schick, M. J. Pol. Sci. B 2003, 41, 1441.

(4) Smit, B.; Hilbers, P. A. J.; Esselink, K.; Rupert, L. A. M.; Van Os, N. M.; Schlijper, A. G. Nature 1990, 348, 624.

(5) Smit, B.; Esselink, K.; Hilbers, P. A. J.; Van Os, N. M.; Rupert, L. A. M.; Szleifer, I. Langmuir 1993, $9,9$.

(6) Palmer, B. J.; Liu, J. Langmuir 1996, 12, 746.

(7) Goetz, R.; Lipowsky, R. J. Chem. Phys. 1998, 108, 7397.

(8) Den Otter, W. K.; Briels, W. J. J. Chem. Phys. 2003, 118, 4712

(9) Von Gottberg, F. K.; Smith, K. A.; Hatton, T. A. J. Chem. Phys. 1997, 106, 9850.

(10) Noguchi, H.; Takasu, M. Phys. Rev. E 2001, 64, 1.

(11) Groot, R. D.; Madden, T. J.; Tildesley, D. J. J. Chem. Phys. 1999 $110,9739$.

(12) Shillcock, J. C.; Lipowsky, R. J. Chem. Phys. 2002, 117, 5048.

(13) Ayton, G.; Voth, G. A. Biophys. J. 2002, 83, 3357.

(14) Venturoli, M.; Smit, B. Phys. Chem. Commun. 1999, 10, 1.

(15) Groot, R. D.; Rabone, K. L. Biophys. J. 2001, 81, 725.

(16) Yamamoto, S.; Maruyama, Y.; Hyodo, S. J. Chem. Phys. 2002, 116,5842

(17) Jury, S.; Bladon, P.; Cates, M.; Krishna, S.; Hagen, M.; Ruddock, N.; Warren, P. Phys. Chem. Chem. Phys. 1999, 1, 2051.

(18) Shelley, J. C.; Shelley, M.; Reeder, R.; Bandyopadhyay, S.; Klein, M. L. J. Phys. Chem. B 2001, 105, 4464.

(19) Lindahl, E.; Hess, B.; Van der Spoel, D. J. Mol. Mod. 2001, 7, 306.

(20) Lide, D. R. CRC Handbook of Chemistry and Physics, 72nd ed.; CRC Press: Boca Raton, FL, 1992.

(21) Douglass, D. C.; Mccall, D. W. J. Phys. Chem. 1958, 62, 1102

(22) Parrinello, M.; Rahman, A. J. Appl. Phys. 1981, 52, 7182.

(23) Schatzberg, P. J. Phys. Chem. 1963, 67, 776.

(24) Ben-Naim, A. Solvation Thermodynamics; Plenum Press: New York, 1987.

(25) Copestake, A.; Neilson, G.; Enderby, J. J. Phys. C: Solid State $1985,18,4211$.

(26) Nagle, J. F.; Tristram-Nagle, S. Biochim. Biophys. Acta 2000, 1469, 159.

(27) Lindahl, E.; Edholm, O. Biophys. J. 2000, 79, 426

(28) Marrink, S. J.; Mark, A. E. J. Phys. Chem. B 2001, 105, 6122

(29) Safran, S. A. Statistical Thermodynamics of Surfaces, Interfaces, and Membranes; Addison-Wesley: Reading, MA, 1994.

(30) Rawicz, W.; Olbrich, K. C.; Mcintosh, T.; Needham, D.; Evans, E. Biophys. J. 2000, 79, 328.

(31) Anézo, C.; de Vries, A. H.; Höltje, H. D.; Tieleman, D. P.; Marrink, S. J. J. Phys. Chem. B 2003, 107, 9424.

(32) Glaser, R. W.; Leikin, S. L.; Chernomordik, L.; Pastushenko, V. F.; Sokirko, A. I. Biochim. Biophys. Acta 1988, 940, 275.

(33) Leontiadou, H.; Mark, A. E.; Marrink, S. J. Biophys. J., in press. 
(34) Chernomordik, L. V.; Kozlov, M. M.; Melikyan, G.; Abidor, I.; Markin, V.; Chizmadzhev, Y. Biochim. Biophys. Acta 1985, 812, 643. 89.

(35) Zhelev, D. V.; Needham, D. Biochim. Biophys. Acta 1993, 1147

(36) Evans, E.; Heinrich, V. Compt. Rend. 2003, 4, 265.

(37) Winterhalter, M. Curr. Opin. Colloid Interface Sc. 2000, 5, 250.

(38) Sheats, J. R.; McConnell, H. M. Proc. Natl. Acad. Sci. U.S.A. 1978, 75, 4661.

(39) Kuo, A. L.; Wade, C. G. Biochemistry 1979, 18, 2300.

(40) Carruthers, A.; Melchior, D. L. Biochemistry 1983, 22, 5797.

(41) Andrasko, J.; Forsen, S. Biochem. Biophys. Res. Commun. 1974, 60,813 .

(42) Nagle, J. F.; Wiener, M. C. Biochim. Biophys. Acta 1988, 942, 1

(43) Balgavý, P.; Dubnicková, M.; Kucerka, N.; Kiselev, M. A.; Yaradaikin, S. P.; Uhríková, D. Biochim. Biophys. Acta 2001, 1512, 40
(44) Petrache, H. I.; Dodd, S.; Brown, M. Biophys. J. 2000, 79, 3172.

(45) Rand, R. P.; Parsegian, V. A. Biochim. Biophys. Acta 1989, 988, 351.

(46) Rand, R. P.; Fuller, N. Biophys. J. 1994, 66, 2127.

(47) Hamm, M.; Kozlov, M. M. Eur. Phys. J. B 1998, 6, 519.

(48) Lauterwein, J.; Bösch, C.; Brown, L. R.; Wüthrich, K. Biochim. Biophys. Acta 1979, 556, 244.

(49) Marrink, S. J.; Tieleman, D. P.; Mark, A. E. J. Phys. Chem. B 2000, 104, 12165 .

(50) Tieleman, D. P.; Van der Spoel, D.; Berendsen, H. J. C. J. Phys. Chem. B 2000, 104, 6380.

(51) Marrink, S. J.; Mark, A. E. J. Am. Chem. Soc., in press.

(52) Marrink, S. J.; Mark, A. E. J. Am. Chem. Soc. 2003, 125, 11144 